\title{
Efficient Randomized Algorithms for Some Geometric Optimization Problems*
}

\author{
P. K. Agarwal ${ }^{1}$ and M. Sharir ${ }^{2}$ \\ ${ }^{1}$ Department of Computer Science, Box 90129, Duke University, \\ Durham, NC 27708-0129, USA \\ pankaj@euclid.cs.duke.edu \\ ${ }^{2}$ School of Mathematical Sciences, Tel Aviv University, \\ Tel Aviv 69978, Israel \\ sharir@math.tau.ac.il \\ and \\ Courant Institute of Mathematical Sciences, New York University, \\ New York, NY 10012, USA
}

\begin{abstract}
In this paper we first prove the following combinatorial bound, concerning the complexity of the vertical decomposition of the minimization diagram of trivariate functions: Let $\mathcal{F}$ be a collection of $n$ totally or partially defined algebraic trivariate functions of constant maximum degree, with the additional property that, for a given pair of functions $f, f^{\prime} \in \mathcal{F}$, the surface $f(x, y, z)=f^{\prime}(x, y . z)$ is $x y$-monotone (actually, we need a somewhat weaker property). We show that the vertical decomposition of the minimization diagram of $\mathcal{F}$ consists of $O\left(n^{3+\varepsilon}\right)$ cells (each of constant description complexity), for any $\varepsilon>0$. In the second part of the paper, we present a general technique that yields faster randomized algorithms for solving a number of geometric optimization problems, including (i) computing the width of a point set in 3-space, (ii) computing the minimum-width annulus enclosing a set of $n$ points in the plane, and (iii) computing the "biggest stick" inside a simple polygon in the plane. Using the above result on vertical decompositions, we show that the expected running time of all three algorithms is $O\left(n^{3 / 2+\varepsilon}\right)$, for any $\varepsilon>0$. Our algorithm improves and simplifies previous solutions of all three problems.
\end{abstract}

\footnotetext{
* Work on this paper by the first author has been supported by NSF Grant CCR-93-01259, an NYI award, and matching funds from Xerox Corporation. Work on this paper by the second author has been supported by NSF Grants CCR-91-22103 and CCR-93-11127, by a Max-Planck Research Award, and by grants from the U.S.-Israeli Binational Science Foundation, the Israel Science Fund administered by the lsraeli Academy of Sciences, and the G.I.F., the German-Israeli Foundation for Scientific Research and Development.
} 


\section{Introduction}

In this paper we present a general technique that yields faster randomized algorithms for the following problems:

1. Computing the width of a set of points in $\mathbb{R}^{3}$.

2. Computing an annulus of minimum width that contains a given set of points in the plane.

3. Computing a longest segment that can be placed inside a simple polygon in the plane.

In order to achieve a fast implementation of our technique, we use the following combinatorial result, which is derived in the first part of the paper. Let $\mathcal{F}$ be a collection of $n$ totally or partially defined algebraic trivariate functions of constant maximum degree, with the following additional $x y$-monotonicity property: For any pair $f, f^{\prime} \in \mathcal{F}$, the $x y$ plane can be decomposed into a constant number of regions, each of constant description complexity, such that, for every region $c$, the surface $f(x, y, z)=f^{\prime}(x, y, z)$ is the graph of a continuous bivariate function (of $x$ and $y$ ) over the interior of $c$. The lower envelope $E_{\mathcal{F}}$ of $\mathcal{F}$ is the pointwise minimum

$$
E_{\mathcal{F}}(x, y, z)=\min _{f \in \mathcal{F}} f(x, y, z),
$$

and the minimization diagram $M_{\mathcal{F}}$ is the projection of the graph of $E_{\mathcal{F}}$ onto $\mathbb{R}^{3}$. That is, $M_{\mathcal{F}}$ is the decomposition of $\mathbb{R}^{3}$ into maximal relatively open connected cells of dimension $0,1,2$, and 3, so that, over each cell, $E_{\mathcal{F}}$ is attained by a fixed subset of functions of $\mathcal{F}$ (and of function boundaries). It is known [29] that $M_{\mathcal{F}}$ has $O\left(n^{3+\varepsilon}\right)$ cells (of all dimensions), for any $\varepsilon>0$.

We prove that the vertical decomposition of $M_{\mathcal{F}}$ also consists of $O\left(n^{3+\varepsilon}\right)$ cells, each of constant description complexity, meaning that it is described by a constant number of polynomial equalities and inequalities of constant maximum degree. See below and [7], [10], [13], and [30] for the definition of vertical decompositions. Briefly, this is the only known general-purpose technique for decomposing cells of arrangements of low-degree algebraic surfaces in higher dimensions into a reasonably small number of subcells of constant description complexity. Such a decomposition is a prerequisite to many randomized incremental or divide-and-conquer algorithms involving arrangements of this kind. Unfortunately, the known upper bounds on the number of resulting subcells are generally much higher than the actual complexity of the cells being decomposed, and this affects adversely (the upper bounds can be proved on) the complexity of the relevant algorithms. Hence any result, like the one we prove here, which establishes nearly tight bounds on the size of vertical decompositions, is significant, as indeed is demonstrated below.

Our bound on the vertical decomposition immediately leads to a data structure, of size $O\left(n^{3+\varepsilon}\right)$, for efficient point-location queries in the region below $E_{\mathcal{F}}$ : For a point $\mathbf{x}=$ $\left(a_{1}, a_{2}, a_{3}, a_{4}\right)$ in $\mathbb{R}^{4}$, we can determine in $O(\log n)$ time whether $a_{4}<E_{\mathcal{F}}\left(a_{1}, a_{2}, a_{3}\right)$. The technique for constructing this data structure crucially relies on the existence of a vertical decomposition of this region (in arrangements of various random samples of $\mathcal{F}$ ) with near-cubic complexity. 
We next observe that each of the three optimization problems mentioned above can be reduced to the problem of computing a closest (or farthest) pair between two sets of objects, under some appropriate (pseudo) distance function. That is, we define two sets of objects $A, B$ and a function $\delta: A \times B \rightarrow \mathbb{R}^{+} \cup\{0\}$, and reduce the original optimization problem to that of computing $\delta^{*}=\min _{a \in A, b \in B} \delta(a, b)$. Actually, we need to solve several instances of the closest-pair problem, but we show that the overall running time is still bounded by (a polylogarithmic factor times) the time complexity of the algorithm for computing $\delta^{*}$. We use a randomized divide-and-conquer approach to compute $\delta^{*}$, which is inspired by the Clarkson-Shor algorithm [11] for computing the diameter of a set of $n$ points in 3-space. The merge and the divide steps of our algorithm require a data structure for point location in the minimization diagram of a set of trivariate functions that satisfy the aforementioned properties. Surprisingly, the $x y$-monotonicity property, which might be regarded as a somewhat restrictive condition, is satisfied for each of the three optimization problems under consideration. Using our bounds on the complexity of vertical decompositions, we show that the expected running time of our algorithms, for all three problems, is $O\left(n^{3 / 2+\varepsilon}\right)$, for any $\varepsilon>0$. The previously best known algorithms for these problems are due to Agarwal et al. [1], and are based on Megiddo's parametric search technique (see also [3] and [8]). The expected running time of the algorithms in [1] is $O\left(n^{17 / 11+\varepsilon}\right)$, for any $\varepsilon>0$.

We consider the main contributions of this paper to be the general algorithmic technique itself and the bound on the size of the vertical decomposition, both of which might be useful in other problems. Even though Megiddo's parametric search technique is a very powerful paradigm, it typically leads to quite complicated algorithms. Recently, there have been several attempts [5], [14], [20], [21], [24] to present simpler and more direct algorithms for some of the problems that have traditionally been solved using parametric searching. Our technique can be viewed as another step in this direction.

The paper is organized as follows. We first establish in Section 2 our bound on the complexity of the vertical decomposition. We then present in Section 3 the general algorithmic technique for computing a closest pair, and exemplify it by applying it to the width problem. We then discuss, more briefly, the minimum-width annulus and the biggest-stick problems in Sections 4 and 5, respectively. Some open problems are mentioned in the concluding Section 6.

\section{Complexity of the Vertical Decomposition}

Let $\mathcal{F}$ be a collection of $n$ totally or partially defined algebraic trivariate functions of constant maximum degree $b$ that satisfy the following properties:

(F1) If a function $f \in \mathcal{F}$ is partially defined, then we require that the set of points where $f$ is undefined have measure zero, in the following strong sense: There is a constant number of algebraic arcs of constant maximum degree in the $x y$ plane (these arcs depend on $f$ ), so that for each point $(x, y)$ not lying on any of these arcs, $f$ is defined at all points $(x, y, z)$, for any $z \in \mathbb{R}$.

(F2) For any pair of functions $f, f^{\prime} \in \mathcal{F}$, the surface $\sigma: f(x, y, z)=f^{\prime}(x, y, z)$ is $x y$-monotone, that is, every $z$-vertical line (not passing through any curve 
where $f$ or $f^{\prime}$ is undefined) crosses this surface in exactly one point. Actually, we relax this assumption somewhat, requiring only that, for each such surface $\sigma$, the $x y$-plane can be decomposed into a constant number of regions, each of constant description complexity, so that, for each of these regions $c$, the surface $\sigma$ is the graph of a continuous bivariate function (of $x$ and $y$ ) over the interior of $c$.

These assumptions are rather restrictive, but as we show below, and rather surprisingly, they hold for several of the current main applications of lower envelopes in 4-space, as listed in the Introduction and studied recently in [1], [3], and [8]. We also assume that the functions in $\mathcal{F}$ are in general position, as defined, e.g., in [29]; it is easy to show, using a variant of the argument given in [29], that this assumption does not involve any loss of generality, and that our results also hold for collections not in general position. Under this assumption, for $j=0, \ldots, 3$, the envelope $E_{\mathcal{F}}$ is attained by at most (or, if the functions in $\mathcal{F}$ are totally defined, exactly) $4-j$ functions of $\mathcal{F}$ over any $j$-dimensional cell of $M_{\mathcal{F}}$. We use the terms vertex, edge, face, and cell to denote, respectively, zero-dimensional, one-dimensional, two-dimensional, and three-dimensional cells of $M_{\mathcal{F}}$. Each vertex, edge, face, or cell $c$ of $M_{\mathcal{F}}$ will be labeled by the corresponding set of functions of $\mathcal{F}$ attaining $E_{\mathcal{F}}$ over $c$. In particular, the term $f$-cell refers to a (three-dimensional) cell of $M_{\mathcal{F}}$ over which $E_{\mathcal{F}}$ is attained by the function $f$ (and only by $f$ ).

The vertical decomposition of $M_{\mathcal{F}}$ is defined in the following standard manner. In the first decomposition stage, we erect, for each edge $e$ of $M_{\mathcal{F}}$, a $z$-vertical wall from $e$, which is the union of all maximal $z$-vertical relatively open segments passing through points of $e$ and not meeting any other vertex, edge, or face of $M_{\mathcal{F}}$. The collection of these walls partitions the cells of $M_{\mathcal{F}}$ into subcells, so that each subcell $c$ is bounded from above and from below (in the $z$-direction) by (portions of ) a fixed pair of faces of $M_{\mathcal{F}} ; c$ may also extend to infinity in either direction. (In general, the situation may be more complicated, but the monotonicity assumption (F2) is easily seen to imply that the structure produced by the first decomposition step does indeed have these properties.) In the second decomposition step, we take each of these subcells $c$ and project it onto the $x y$-plane. We construct the two-dimensional vertical decomposition of the projection $c^{*}$, by erecting a maximal $y$-vertical segment, contained in the closure of $c^{*}$, from each vertex of $c^{*}$ and each (locally) $x$-extremal point on $\partial c^{*}$. The collection of these segments partitions $c^{*}$ into "pseudo-trapezoidal" subcells. Each of these subcells $\tau$ induces a subcell of $c$, obtained by intersecting $c$ with the vertical cylinder $\tau \times \mathbb{R}$ over $\tau$. (Again, assumption (F2) implies that $\tau$ induces only one subcell of $c$.) The resulting collection of subcells constitutes the vertical decomposition of $M_{\mathcal{F}}$, which we denote by $M_{\mathcal{F}}^{*}$. Each of these cells has constant description complexity, meaning, as above, that it is defined by a constant number of polynomial equalities and inequalities of constant maximum degree (depending on the maximum degree $b$ of the functions of $\mathcal{F}$ ). See [7], [10], and [30] for more details concerning vertical decompositions.

Theorem 2.1. If $\mathcal{F}$ is a collection of trivariate functions satisfying the assumptions made above, then the number of subcells of $M_{\mathcal{F}}^{*}$ is $O\left(n^{3+\varepsilon}\right)$, for any $\varepsilon>0$, where the constant of proportionality depends on $\varepsilon$ and on the maximum degree $b$. 
Proof. Let $\mathcal{F}$ be a collection of $n$ trivariate functions satisfying the above assumptions. It is easily seen that, in general, the second vertical decomposition step does not increase the complexity of the decomposition by more than a constant factor, so it suffices to bound the increase in the complexity of $M_{\mathcal{F}}$ caused by the first vertical decomposition step. In other words, we want to count the number of pairs $\left(e, e^{t}\right)$ of edges of $M_{\mathcal{F}}$, both bounding the same cell $c$, for which there exists a $z$-vertical segment connecting a point on $e$ to a point on $e^{\prime}$ and fully contained in $c$. (We actually want to count the number of these vertical segments, but, by assumption, this number is larger than the number of pairs $\left(e, e^{\prime}\right)$ by only a constant factor, depending on the maximum degree $b$.) We say that such a pair $\left(e, e^{\prime}\right)$ of edges are vertically visible. Suppose $c$ is an $f_{0}$-cell, for some $f_{0} \in \mathcal{F}$ (note that assumption (F1) implies that there is no three-dimensional cell of $M_{\mathcal{F}}$ over which $E_{\mathcal{F}}$ is undefined). Then $e$ must be either a portion of an intersection curve of the form $f_{0}=f_{1}=f_{2}$, for some pair of functions $f_{1}, f_{2} \in \mathcal{F}$, or a portion of the boundary of an $x y$-monotone piece of a surface $f_{0}=f$, for some $f \in \mathcal{F}$. Similarly, $\boldsymbol{e}^{\prime}$ must also be a portion of an intersection curve or of a boundary curve of the above forms.

We estimate the number of vertically visible pairs of edges for which the vertical segment connecting the edges crosses an $f_{0}$-cell, separately for each fixed $f_{0} \in \mathcal{F}$. Recall that, by assumption (F1), each surface $f_{0}=f$ can be decomposed into a constant number of $x y$-monotone pieces, so that the $x y$-projections of these pieces are pairwise disjoint. Consider the collection $\Sigma\left(f_{0}\right)$ consisting of all these $x y$-monotone portions of surfaces of the form $f_{0}=f$, for $f \in \mathcal{F}$. We regard each such portion as a partially defined function of $x$ and $y$. It follows from assumption (F2) that, for each surface $\sigma \in \Sigma\left(f_{0}\right)$, contained in the graph of $f_{0}=f$, for some $f \in \mathcal{F}$, either all points lying vertically above $\sigma$ (in the $z$-direction) satisfy $f_{0}>f$ or all such points satisfy $f_{0}<f$ (this property may fail at points lying on the boundary $\partial \sigma$ of $\sigma$, but this limit behavior does not affect our analysis). Moreover, by the general position assumption, the reverse inequalities hold at points lying vertically below $\sigma$. Let $\Sigma^{+}\left(f_{0}\right)$ (resp. $\Sigma^{-}\left(f_{0}\right)$ ) denote the subset of surfaces $\sigma \in \Sigma\left(f_{0}\right)$ for which the corresponding function $f$ satisfies $f_{0}>f$ (resp. $f_{0}<f$ ) for all points lying vertically above $\sigma$ (note that a function $f$ may contribute surfaces to both collections $\Sigma^{+}\left(f_{0}\right), \Sigma^{-}\left(f_{0}\right)$, over pairwise-disjoint portions of the $x y$-plane). It is then clear that the union of all $f_{0}$-cells is the same as the region enclosed between the upper envelope of $\Sigma^{-}\left(f_{0}\right)$ and the lower envelope of $\Sigma^{+}\left(f_{0}\right)$. It then follows from Theorem 3.2 of [2], concerning the complexity of the region enclosed between two envelopes in 3 -space, that the complexity of the vertical decomposition of all $f_{0}$-cells is $O\left(n^{2+\varepsilon}\right)$, for any $\varepsilon>0$. Repeating this argument over all functions $f_{0} \in \mathcal{F}$, we obtain the bound asserted in the theorem.

Let $C_{\mathcal{F}}$ be the cell in the arrangement of $\mathcal{F}$ lying below $E_{\mathcal{F}}$. The vertical decomposition of $C_{\mathcal{F}}$, denoted as $C_{\mathcal{F}}^{*}$, can be obtained by lifting each cell $\tau \in M_{\mathcal{F}}^{*}$ to the cell

$$
\hat{\tau}=\left\{(\mathbf{x}, z) \mid \mathbf{x} \in \tau,-\infty<z \leq E_{\mathcal{F}}(\mathbf{x})\right\} .
$$

Since each cell of $M_{\mathcal{F}}^{*}$ contributes exactly one cell to $C_{\mathcal{F}}^{*}$, the latter also has $O\left(n^{3+\varepsilon}\right)$ cells. Similarly, it follows that the vertical decomposition of the cell lying above the graphs of all functions in $\mathcal{F}$ also has $O\left(n^{3+\varepsilon}\right)$ cells, for any $\varepsilon>0$. 
Remark 2.2. An obvious open problem is to extend this bound to vertical decompositions of minimization diagrams of more general trivariate functions. Using recent analysis techniques, as those in [2] and [29], we can obtain an $O\left(n^{4+\varepsilon}\right)$ bound for the general case of partially defined trivariate low-degree algebraic functions, but we conjecture that the correct bound is near-cubic, as above.

\section{Width in 3-Space}

The width of a set $S$ of $n$ points in $\mathbb{R}^{3}$ is the smallest distance between a pair of parallel planes such that the closed slab between the planes contains $S$. Although the width of a set of $n$ points in the plane can be computed in $O(n \log n)$ time [19], the problem is considerably harder in three dimensions. Houle and Toussaint [19] gave a simple $O\left(n^{2}\right)$ time algorithm for computing the width in $\mathbb{R}^{3}$, and raised the open problem of obtaining a subquadratic solution. Chazelle et al. [8] presented an $O\left(n^{8 / 5+\varepsilon}\right)$-time algorithm, for any $\varepsilon>0$, which was subsequently improved by Agarwal et al. [1] to $O\left(n^{17 / 11+\varepsilon}\right)$. In this section we present a randomized algorithm whose expected running time is $O\left(n^{3 / 2+\varepsilon}\right)$. For the sake of simplicity, we assume that the points in $S$ are in general position so that no edge of the convex hull of $S$ is parallel to the $y z$-plane, no two edges of the convex hull are parallel, etc.

As observed in [8], and further exploited in [1], the problem of computing the width in 3-space can be reduced to the following bichromatic closest line-pair problem: Given a set $L$ of $m$ "red" lines and another set $L^{\prime}$ of $n$ "blue" lines in $\mathbb{R}^{3}$, such that no line lies parallel to the $y z$-plane and no two of them are parallel to each other, all red lines lie above all blue lines, ${ }^{1}$ compute the closest pair of lines in $L \times L^{\prime}$, where the distance between a pair of lines $\ell, \ell^{\prime} \in \mathbb{R}^{3}$ is

$$
d\left(\ell, \ell^{\prime}\right)=\min _{p \in \ell, q \in \ell^{\prime}} d(p, q),
$$

where $d(p, q)$ is the Euclidean distance in $\mathbb{R}^{3}$. Let $d\left(L, L^{\prime}\right)=\min _{\ell \in L, \ell^{\prime} \in L^{\prime}} d\left(\ell, \ell^{\prime}\right)$ denote the distance between a closest pair in $L \times L^{\prime}$.

Before presenting the algorithm, we need to describe some geometric transforms, which are crucial for our algorithm. We can map each line $\ell$ in 3-space, not parallel to the $y z$-plane, to a point $\psi(\ell)=\left(a_{1}, a_{2}, a_{3}, a_{4}\right)$ in $\mathbb{R}^{4}$, where $y=a_{1} x+a_{3}$ is the equation of the $x y$-projection of $\ell$, and $z=a_{2} x+a_{4}$ is the equation of the $x z$-projection of $\ell$. For any fixed real parameter $\delta \geq 0$, we can also map a line $\ell^{\prime}$ in 3-space to a surface $\gamma\left(\ell^{\prime}\right)$, which is the locus of all points $\psi(\ell)$ such that $d\left(\ell, \ell^{\prime}\right)=\delta$ and $\ell$ lies above $\ell^{\prime}$. We refer to the coordinates of this parametric space as $\xi_{1}, \xi_{2}, \xi_{3}, \xi_{4}$. Observe that any line $\lambda$ parallel to the $\xi_{4}$-axis intersects $\gamma=\gamma\left(\ell^{\prime}\right)$ in at most one point (this is because any two lines in $\mathbb{R}^{3}$ mapped into points on $\lambda$ are translates of each other in the $z$-direction). If the lines $\ell$ in $\mathbb{R}^{3}$ that are mapped to $\lambda$ lie in a vertical plane parallel to $\ell^{\prime}$ and not containing

\footnotetext{
${ }^{1}$ For any pair of nonparallel and nonvertical lines $\ell \in L, \ell^{\prime} \in L^{\prime}$, we say that $\ell$ lies above $\ell^{\prime}$ if the vertical line passing through the intersection point of the $x y$-projections of $\ell$ and $\ell^{\prime}$ intersects $\ell$ above $\ell^{\prime}$. It is interesting to note that the requirement that all red lines lie above all blue lines crucially affects the analysis of the complexity of the resulting algorithm.
} 
$\ell^{\prime}$, then $\lambda$ may fail to intersect $\gamma$. It follows that $\gamma$ can be partitioned into a constant number of surface patches, each of constant description complexity, so that for each patch $\tilde{\gamma}$, all points of $\mathbb{R}^{4}$ lying vertically above $\tilde{\gamma}$ represent lines $\ell$ in $\mathbb{R}^{3}$ that lie above $\ell^{\prime}$ and $d\left(\ell, \ell^{\prime}\right)>\delta$, and all points lying below $\tilde{\gamma}$ represent lines $\ell$ that either lie below $\ell^{\prime}$, pass through $\ell^{\prime}$, or lie above $\ell^{\prime}$ and $d\left(\ell, \ell^{\prime}\right)<\delta$. In other words, $\gamma\left(\ell^{\prime}\right)$ is the graph of a partially defined function $\xi_{4}=f_{\ell^{\prime}}\left(\xi_{1}, \xi_{2}, \xi_{3}\right)$. For a point $\psi(\ell)=\left(a_{1}, a_{2}, a_{3}, a_{4}\right)$, such that $\ell$ lies above $\ell^{\prime}$, if $f_{\ell^{\prime}}\left(a_{1}, a_{2}, a_{3}\right)$ is defined, then $a_{4}>f_{\ell^{\prime}}\left(a_{1}, a_{2}, a_{3}\right)$ if and only if $d\left(\ell, \ell^{\prime}\right)>\delta$, and $a_{4}<f_{\ell^{\prime}}\left(a_{1}, a_{2}, a_{3}\right)$ if and only if $d\left(\ell, \ell^{\prime}\right)<\delta$. Let $\mathcal{F}$ be the collection $\left\{f_{\ell^{\prime}} \mid \ell^{\prime} \in L^{\prime}\right\}$, and let $U_{\mathcal{F}}$ denote the upper envelope of $\mathcal{F}$. For a line $\ell \in L$ with $\psi(\ell)=\left(a_{1}, a_{2}, a_{3}, a_{4}\right)$, we have $a_{4} \geq U_{\mathcal{F}}\left(a_{1}, a_{2}, a_{3}\right)$ if and only if $d\left(\{\ell\}, L^{\prime}\right) \geq \delta$. It is easily checked that the functions $f_{\ell^{\prime}}$ are all partially defined algebraic functions of constant maximum degree.

\section{Lemma 3.1.}

(a) For any line $\ell^{\prime} \in L^{\prime}$ and for any fixed $\xi_{1}, \xi_{2}$, so that $\xi_{1}$ is not equal to the $\xi_{1}$-coordinate of $\ell^{\prime}$, the function $f_{\ell^{\prime}}\left(\xi_{1}, \xi_{2}, \xi_{3}\right)$ is defined for all $\xi_{3}$.

(b) For any pair of nonparallel lines $\ell_{1}^{\prime}, \ell_{2}^{\prime} \in L^{\prime}$ and for any fixed $\xi_{1}, \xi_{2}$, the equation

$$
f_{\ell_{1}^{\prime}}\left(\xi_{1}, \xi_{2}, \xi_{3}\right)=f_{\ell_{2}^{\prime}}\left(\xi_{1}, \xi_{2}, \xi_{3}\right)
$$

has a unique solution $\xi_{3}$, except when $\left(\xi_{1}, \xi_{2}\right)$ lies on a certain critical line $\lambda\left(\ell_{1}^{\prime}, \ell_{2}^{\prime}\right)$ that depends on $\ell_{1}^{\prime}$ and $\ell_{2}^{\prime}$, or when $\xi_{1}$ is equal to the $\xi_{1}$-coordinate of $\ell_{1}^{\prime}$ or of $\ell_{2}^{\prime}$.

Proof. Part (a) is trivial: Let $\xi_{1}, \xi_{2}, \xi_{3}$ be a triple such that $\xi_{1}$ is not equal to the $\xi_{1}$ coordinate of $\ell^{\prime}$. Then the spatial orientation of all corresponding lines is fixed and their $x y$-projection has a different orientation than that of $\ell^{\prime}$. Consider the line of the form $y=\xi_{1} x+\xi_{3}, z=\xi_{2} x+\xi_{4}$ which intersects $\ell^{\prime}$. If we translate this line in the $+z-$ direction (i.e., increase the value of $\xi_{4}$ ), its distance from $\ell^{\prime}$ monotonically increases (it actually varies linearly in $\xi_{4}$, as is easily checked), and therefore there is a unique $\xi_{4}=\alpha$ such that the line $\ell$ with $\varphi(\ell)=\left(\xi_{1}, \xi_{2}, \xi_{3}, \alpha\right)$ lies above $\ell^{\prime}$ and $d\left(\ell, \ell^{\prime}\right)=\delta$. Hence, $f_{\ell^{\prime}}\left(\xi_{1}, \xi_{2}, \xi_{3}\right)$ is defined. (If the $x y$-projections of these lines have the same orientation as that of $\ell^{\prime}$, then $f_{\ell^{\prime}}\left(\xi_{1}, \xi_{2}, \xi_{3}\right)$ is defined only when $\xi_{2}$ is equal to the $\xi_{2}$-coordinate of $\ell^{\prime}$ and when $\xi_{3}$ is such that $\ell$ and $\ell^{\prime}$ lie in the same vertical plane.)

Consider next part (b). Let $\xi_{3}$ be a solution of (3.1), and let $\xi_{4}=f_{\ell_{1}^{\prime}}\left(\xi_{1}, \xi_{2}, \xi_{3}\right)=$ $f_{\ell_{2}^{\prime}}\left(\xi_{1}, \xi_{2}, \xi_{3}\right)$. The line $\ell^{*}$, parametrized by $\left(\xi_{1}, \xi_{2}, \xi_{3}, \xi_{4}\right)$, thus lies in the vertical plane $\pi\left(\xi_{3}\right): y=\xi_{1} x+\xi_{3}$, and, as is easily checked, its slope in that plane, with respect to the coordinate frame $(u, z)$, where $u$ is the axis orthogonal to the $z$-axis, is equal to $\xi_{2} / \sqrt{1+\xi_{1}^{2}}$. Moreover, by definition, $\ell^{*}$ is a common upper tangent line to the two cylinders $C_{1}, C_{2}$ of radius $\delta$, whose symmetry axes are the lines $\ell_{1}^{\prime}, \ell_{2}^{\prime}$, respectively. Let $K_{i}=K_{i}\left(\xi_{3}\right)=C_{i} \cap \pi\left(\xi_{3}\right)$, for $i=1,2$. The sets $K_{1}$ and $K_{2}$ are two ellipses, and the line $\ell^{*}$ must be a common upper tangent to $K_{1}$ and $K_{2}$ in the plane $\pi\left(\xi_{3}\right)$ (this holds provided that $\xi_{1}$ is not equal to the $\xi_{1}$-coordinate of $\ell_{1}^{\prime}$ or of $\left.\ell_{2}^{\prime}\right)$. As $\xi_{3}$ varies, the plane $\pi\left(\xi_{3}\right)$ translates parallel to itself, and the two ellipses $K_{i}\left(\xi_{3}\right)$ also translate within that plane, so that the positions of their centers are given by two linear functions of $\xi_{3}$. Moreover, 
for $i=1,2$, let $w_{i}=w_{i}\left(\xi_{3}\right)$ denote the point on $K_{i}\left(\xi_{3}\right)$ so that the line tangent to $K_{i}$ at $w_{i}$ has slope $\xi_{2} / \sqrt{1+\xi_{1}^{2}}$ and lies above $K_{i}$. It follows that, as $\xi_{3}$ varies, $w_{i}\left(\xi_{3}\right)$ moves within the plane $\pi\left(\xi_{3}\right)$ as a linear function of $\xi_{3}$, for $i=1,2$. Thus, $\xi_{3}$ solves (3.1) if and only if the line connecting $w_{1}\left(\xi_{3}\right)$ and $w_{2}\left(\xi_{3}\right)$ has slope $\xi_{2} / \sqrt{1+\xi_{1}^{2}}$ in $\pi\left(\xi_{3}\right)$. This equation is linear in $\xi_{3}$, as easily follows from the above arguments, and so has one solution, no solutions, or infinitely many solutions.

To analyze when this equation has no solution, or has infinitely many solutions, we represent the above geometric reasoning in an algebraic form. It is easily verified that the existence of a unique solution to (3.1) is not affected if we translate $\ell_{1}^{\prime}$ and $\ell_{2}^{\prime}$ by any amounts (such a translation only changes the constant term in the resulting linear equation), so we may assume, with no loss of generality, that both lines pass through the origin. Let $\left(a_{1}, b_{1}, c_{1}\right),\left(a_{2}, b_{2}, c_{2}\right)$ be two unit vectors lying, respectively, on the lines $\ell_{1}^{\prime}, \ell_{2}^{\prime}$. The intersection $s_{1}\left(\xi_{3}\right)$ of $\ell_{1}^{\prime}$ with $\pi\left(\xi_{3}\right)$ is a point $\left(a_{1} t, b_{1} t, c_{1} t\right)$ that satisfies the equation $b_{1} t=\xi_{1} a_{1} t+\xi_{3}$, so we have $t=\xi_{3} /\left(b_{1}-a_{1} \xi_{1}\right)$, which implies that $s_{1}\left(\xi_{3}\right)$ is the point

$$
\left(\frac{a_{1} \xi_{3}}{b_{1}-a_{1} \xi_{1}}, \frac{b_{1} \xi_{3}}{b_{1}-a_{1} \xi_{1}}, \frac{c_{1} \xi_{3}}{b_{1}-a_{1} \xi_{1}}\right)
$$

and, similarly, the intersection $s_{2}\left(\xi_{3}\right)$ of $\ell_{2}^{\prime}$ with $\pi\left(\xi_{3}\right)$ is the point

$$
\left(\frac{a_{2} \xi_{3}}{b_{2}-a_{2} \xi_{1}}, \frac{b_{2} \xi_{3}}{b_{2}-a_{2} \xi_{1}}, \frac{c_{2} \xi_{3}}{b_{2}-a_{2} \xi_{1}}\right) \text {. }
$$

(As above, these points are well defined only when $\xi_{1}$ is not equal to the $\xi_{1}$-coordinate of $\ell_{1}^{\prime}$ or of $\ell_{2}^{\prime}$.) For $i=1,2$, the point $w_{i}\left(\xi_{3}\right)$ is a translated copy of $s_{i}\left(\xi_{3}\right)$ by a fixed vector, independent of $\xi_{3}$. The coefficient of $\xi_{3}$ in (3.1) is thus easily seen to be (proportional to)

$$
\frac{c_{2}}{b_{2}-a_{2} \xi_{1}}-\frac{c_{1}}{b_{1}-a_{1} \xi_{1}}-\xi_{2}\left(\frac{a_{2}}{b_{2}-a_{2} \xi_{1}}-\frac{a_{1}}{b_{1}-a_{1} \xi_{1}}\right) \text {. }
$$

Hence, (3.1) does not have a unique solution only when this expression is zero. That is,

$$
\frac{c_{2}-a_{2} \xi_{2}}{b_{2}-a_{2} \xi_{1}}=\frac{c_{1}-a_{1} \xi_{2}}{b_{1}-a_{1} \xi_{1}}
$$

which is easily seen to be a linear equation in $\xi_{1}$ and $\xi_{2}$ (it does not vanish identically, unless $\ell_{1}^{\prime}$ and $\ell_{2}^{\prime}$ are parallel). This completes the proof of the lemma.

Lemma 3.1 implies that the collection $\mathcal{F}$ satisfies the assumptions (F1), (F2) of Theorem 2.1. Let $C_{\mathcal{F}}$ denote the cell in the arrangement of $\mathcal{F}$ that lies above the upper envelope of $\mathcal{F}$. In view of Lemma 3.1, Theorem 2.1, the above discussion, and standard point-location techniques that are based on vertical decompositions (such as those in [7] and [9]), we obtain:

Corollary 3.2. The vertical decomposition $C_{\mathcal{F}}^{*}$ of $C_{\mathcal{F}}$ consists of $O\left(n^{3+\varepsilon}\right)$ cells, for any $\varepsilon>0$. Moreover, $C_{\mathcal{F}}$ can be preprocessed in time $O\left(n^{3+\varepsilon}\right)$ into a data structure of size $O\left(n^{3+\varepsilon}\right)$, for any $\varepsilon>0$, so that, for any query point $p$, we can determine in $O(\log n)$ time whether $p \in C_{\mathcal{F}}$. 
We now describe a divide-and-conquer algorithm for computing $d\left(L, L^{\prime}\right)$. The basic idea is as follows: We randomly choose a line $\ell \in L$, and compute the distance $\delta_{0}$ between $\ell$ and its nearest neighbor in $L^{\prime}$. We discard all those lines of $L$ whose nearest neighbors in $L$ are at distance $\geq \delta_{0}$. Let $L_{1} \subset L$ be the subset of remaining lines. If $L_{1}=\emptyset$, we return $\delta_{0}$; otherwise, we recursively compute $d\left(L_{1}, L^{\prime}\right)$. Using a probabilistic argument, it can be shown that the expected depth of the recursion is $O(\log m)$. The only nontrivial step in the above algorithm is computing the subset $L_{1}$. However, we do not have an efficient procedure for computing $L_{1}$, so, after computing $\delta_{0}$, we proceed in a roundabout way. We divide the problem of computing $d\left(L, L^{\prime}\right)$ into a number of subproblems, of which one is solved recursively and the others are solved using a different algorithm, as described below. We first present an outline of the algorithm, and then explain each of the nontrivial steps in more detail.

\section{ALgORITHM ClOSEST-PAIR}

The input consists of two sets, $L, L^{\prime}$, of lines in 3-space satisfying the above assumptions, with $|L|=m$ and $\left|L^{\prime}\right|=n$ :

1. Let $n_{0}$ be a sufficiently large constant, whose value will be fixed later. If $n \leq n_{0}$, then we compute $d\left(\ell, \ell^{\prime}\right)$ for every pair $\left(\ell, \ell^{\prime}\right) \in L \times L^{\prime}$, in $O(m)$ time, and return the minimum distance.

2. Assume that $n>n_{0}$. Randomly choose a line $\ell_{0} \in L$ and compute $\delta_{0}=$ $d\left(\left\{\ell_{0}\right\}, L^{\prime}\right)$, in $O(n)$ time.

3. Set $r=\left\lceil m^{3 / 8} / n^{1 / 8}\right\rceil$. We partition $L$ into $k+1$ subsets $L_{0}, L_{1}, \ldots, L_{k}$, with the following properties:

(i) $k=O\left(r^{3+\varepsilon}\right)$, for any $\varepsilon>0$;

(ii) if $r=1$, then $k=1, L_{0}=\emptyset, L_{\mathrm{l}}=L$;

(iii) for each $1 \leq i \leq k$,

$$
\left|\left\{\ell^{\prime} \in L^{\prime} \mid d\left(L_{i},\left\{\ell^{\prime}\right\}\right)<\delta_{0}\right\}\right| \leq \frac{n}{r}
$$

(iv) $L_{0} \subseteq\left\{\ell \in L \mid d\left(\{\ell\}, L^{\prime}\right)<\delta_{0}\right\} ; L_{0}$ may be empty (as is the case when $r=1$ ).

4. For each $1 \leq i \leq k$, we compute a set $L_{i}^{\prime}$ of size at most $n / r$ such that

$$
L_{i}^{\prime} \supseteq\left\{\ell^{\prime} \in L^{\prime} \mid d\left(L_{i},\left\{\ell^{\prime}\right\}\right)<\delta_{0}\right\}
$$

if $r=1$ (and $k=1$ ), we put $L_{1}^{\prime}=L^{\prime}$. Set $m_{i}=\left|L_{i}\right|$ and $n_{i}=\left|L_{i}^{\prime}\right|$.

5. For each $1 \leq i \leq k$, we do the following: If $n_{i}=0$, we set $d\left(L_{i}, L_{i}^{\prime}\right)=+\infty$. Otherwise, we compute $\delta_{i}^{*}=d\left(L_{i}, L_{i}^{\prime}\right)$ directly, using a different algorithm (detailed below). We then compute $\delta_{1}=\min _{i} \delta_{i}^{*}$.

6. If $L_{0} \neq \emptyset$, we compute $\delta_{2}=d\left(L_{0}, L^{\prime}\right)$ recursively; otherwise, put $\delta_{2}=+\infty$.

7. Return $\min \left\{\delta_{0}, \delta_{1}, \delta_{2}\right\}$ as $d\left(L, L^{\prime}\right)$.

Next, we explain Steps 3-5 in detail, and analyze their expected running time; the other steps are trivial and need no further explanation. We then conclude the analysis by proving the correctness of the algorithm. 
Steps 3 and 4 . We compute $L_{i}, L_{i}^{\prime}$, for $1 \leq i \leq k$, using a divide-and-conquer approach. We construct a tree $T$, each of whose nodes $v$ is associated with two subsets $L_{v} \subseteq L$ and $L_{v}^{\prime} \subseteq L^{\prime}$. The root of the tree is associated with $L$ and $L^{\prime}$ themselves. The subsets associated with the leaves of $T$ correspond to the sets $L_{i}$ and $L_{i}^{\prime}$. The set $L_{0}$ consists of those lines that are not passed down the recursive construction of $T$.

If $r=1$, then $T$ consists of a single node; we set $k=1, L_{1}=L, L_{1}^{\prime}=L^{\prime}$, and $L_{0}=\emptyset$. Next, assume that $r>1$. Let $s$ be some sufficiently large constant. We choose a random subset $X \subseteq L^{\prime}$ of size $c_{1} s \log s$, where $c_{1}$ is an appropriate constant independent of $s$, and compute $C_{X}^{*}$, the vertical decomposition of the cell $C_{X}$ lying above the graphs of all the functions $\left\{f_{\ell^{\prime}} \mid \ell^{\prime} \in X\right\}$ (defined in terms of the parameter $\delta_{0}$ computed in Step 2). By Corollary 3.2, $C_{X}^{*}$ has $O\left((s \log s)^{3+\varepsilon}\right)$ cells, for any $\varepsilon>0$. For each cell $\tau \in C_{X}^{*}$, we compute the set $L_{\tau}^{\prime} \subseteq L^{\prime}$ of lines $\ell^{\prime}$ such that $\gamma\left(\ell^{\prime}\right)$ intersects $\tau$. By standard $\varepsilon$-net theory [18], we have, with high probability, $\left|L_{\tau}^{\prime}\right| \leq n / s$ for every $\tau \in C_{X}^{*}$. If $\left|L_{\tau}^{\prime}\right|>n / s$ for some $\tau \in C_{X}^{*}$, we discard $X$, choose another random subset, and repeat the above steps. Otherwise, for each $\tau \in C_{X}^{*}$ we compute the subset $L_{\tau} \subseteq L$ of lines $\ell$ such that $\psi(\ell) \in \tau$. Set $m_{\tau}=\left|L_{\tau}\right|$ and $n_{\tau}=\left|L_{\tau}^{\prime}\right|$. If $L_{\tau} \neq \emptyset$, we create a child $v_{\tau}$ of the root of $T$ corresponding to $\tau$. We associate $L_{\tau}, L_{\tau}^{\prime}$ with $v_{\tau}$. If $\left|L_{\tau}^{\prime}\right| \leq n / r$, then $v_{\tau}$ is a leaf. Otherwise, $v_{\tau}$ is an internal node of $T$, and we expand $T$ further at $v_{\tau}$ by applying the same procedure recursively to $L_{\tau}, L_{\tau}^{\prime}$ (using the same constant $s$ ).

By construction, the depth of $T$ is at most $\left\lceil\log _{s} r\right\rceil$. Since each node has at most $O\left((s \log s)^{3+\varepsilon}\right)$ children, the total number of leaves in $T$ is $k \leq c_{2} r^{3+\varepsilon^{\prime}}$, where $\varepsilon^{\prime}=$ $\varepsilon+O(\log \log s / \log s)$ and where $c_{2}$ is a constant depending on $\varepsilon^{\prime}$ (and thus on $\varepsilon$ and $s) ; \varepsilon^{\prime}$ can be made arbitrarily close to $\varepsilon$ by choosing $s$ to be sufficiently large. We set $L_{i}$ and $L_{i}^{\prime}$ to be the subsets associated with the $i$ th leaf of $T$, for $i=1, \ldots, k$. Finally, we set $L_{0}=L-\bigcup_{i=1}^{k} L_{i}$. Note that a line $\ell$ is placed in $L_{0}$ only when its image $\psi(\ell)$ lies below the upper envelope of some collection $\left\{f_{\ell^{\prime}} \mid \ell^{\prime} \in X\right\}$, for some $X \subseteq L^{\prime}$. Hence, by definition, all lines $\ell \in L_{0}$ satisfy $d\left(\{\ell\}, L^{\prime}\right)<\delta_{0}$. In particular, $\ell_{0} \notin L_{0}$, so $\left|L_{0}\right|<m$, a property that we use below when proving the correctness of the algorithm. This also shows that $L_{0}$ satisfies property (iv) of Step 3 .

The sets $L_{i}$, for $1 \leq i \leq k$, are pairwise disjoint, and $\left|L_{i}^{\prime}\right| \leq n / r$, for all $1 \leq i \leq k$. It thus remains to show that

$$
L_{i}^{\prime} \supseteq\left\{\ell^{\prime} \in L^{\prime} \mid d\left(\left\{\ell^{\prime}\right\}, L_{i}\right)<\delta_{0}\right\}
$$

In fact, the following stronger claim is true, and follows easily by construction.

Lemma 3.3. For any node $v_{\tau}$ in $T$,

$$
L_{\tau}^{\prime} \supseteq L_{\tau}^{\prime \prime}=\left\{\ell^{\prime} \in L^{\prime} \mid d\left(\left\{\ell^{\prime}\right\}, L_{\tau}\right)<\delta_{0}\right\} .
$$

Proof. We prove this by induction on the depth of $v_{\tau}$ in $T$. The claim obviously holds for the root of $T$. Suppose it holds for the parent $v_{\zeta}$ of a node $v_{\tau}$. Since $L_{\tau} \subseteq L_{\zeta}$, obviously $L_{\tau}^{\prime \prime} \subseteq L_{\zeta}^{\prime \prime}$. Let $C_{X}^{*}$ be the set of cells that were constructed at $v_{\zeta}$. Then $\tau \in C_{X}^{*}$ and, for every $\ell \in L_{\tau}$, we have $\psi(\ell) \in \tau$. Let $\ell^{\prime} \in L_{\tau}^{\prime \prime}$ and let $\ell$ be a line in $L_{\tau}$ satisfying $d\left(\ell, \ell^{\prime}\right)<\delta_{0}$. Then, by definition, the point $\psi(\ell)$ lies below the surface $\gamma\left(\ell^{\prime}\right)$. Since $\tau$ is unbounded in the $+\xi_{4}$-direction, it follows that $\gamma\left(\ell^{\prime}\right)$ intersects $\tau$. Moreover, by the 
induction hypothesis, $\ell^{\prime} \in L_{\zeta}^{\prime}$, which implies that $\ell \in L_{\tau}^{\prime}$, and thus the claim is true for $\tau$ as well.

Hence, the sets $L_{i}, L_{i}^{\prime}$, for $1 \leq i \leq k$, satisfy the desired properties of Steps 3 and 4 .

Next, we analyze the expected time spent in computing these subsets. Let $f(a, b)$ denote the maximum expected time spent by the recursive algorithm for Steps 3 and 4 , where expectation is with respect to the choices of random samples by the algorithm, and where the maximum is taken over all sets $L, L^{\prime}$ of lines, as above, of respective sizes $a, b$. At each level of recursion, the expected number of choices of $X$ is a constant, and we spend $O\left((s \log s)^{3+\varepsilon}(a+b)\right)$ time to compute all the sets $L_{\tau}, L_{\tau}^{\prime}$. Since $C_{X}^{*}$ consists of $O\left((s \log s)^{3+\varepsilon}\right)$ cells, we obtain the following recurrence:

$$
f(a, b) \leq \sum_{i=1}^{c(s \log s)^{3+\varepsilon}} f\left(a_{i}, b_{i}\right)+c^{\prime}(s \log s)^{3+\varepsilon}(a+b),
$$

where $\sum_{i} a_{i} \leq a, b_{i} \leq b / s$, and $c, c^{\prime}$ are constants (depending on $\varepsilon$ ). The recursion stops when $b \leq n / r$, so $f(a, b)=O(1)$ for $b \leq n / r$.

The solution of the above recurrence is

$$
f(a, b) \leq A\left(a \log b+\frac{b^{3+\varepsilon^{\prime}}}{n^{2}} r^{2}\right),
$$

for any $\varepsilon^{\prime}>\varepsilon$; here $A=A\left(\varepsilon^{\prime}\right)$ is a sufficiently large constant depending on $\varepsilon$.

We prove this by induction on $b$ (considering $n$ to be a fixed parameter). The inequality obviously holds for $b \leq n / r$. For larger values of $b$, we obtain, by the induction hypothesis,

$$
\begin{aligned}
f(a, b) \leq & \sum_{i=1}^{c(s \log s)^{3+\varepsilon}} A\left(a_{i} \log b_{i}+\frac{b_{i}^{3+\varepsilon^{\prime}}}{n^{2}} r^{2}\right)+c^{\prime}(s \log s)^{3+\varepsilon}(a+b) \\
\leq & A \sum_{i=1}^{c(s \log s)^{3+\varepsilon}}\left(a_{i} \log \frac{b}{s}+\left(\frac{b}{s}\right)^{3+\varepsilon^{\prime}} \cdot \frac{r^{2}}{n^{2}}\right)+c^{\prime}(s \log s)^{3+\varepsilon}(a+b) \\
\leq & A a \log b+a\left[c^{\prime}(s \log s)^{3+\varepsilon}-A \log s\right] \\
& +A b^{3+\varepsilon^{\prime}} \frac{r^{2}}{n^{2}}\left[c s^{\varepsilon-\varepsilon^{\prime}} \log ^{3+\varepsilon} s+\frac{c^{\prime}}{A} \frac{n^{2} / r^{2}}{b^{2}}(s \log s)^{3+\varepsilon}\right] \\
\leq & A\left(a \log b+b^{3+\varepsilon^{\prime}} \frac{r^{2}}{n^{2}}\right) .
\end{aligned}
$$

The last inequality holds because $b>n / r$ and $\varepsilon^{\prime}>\varepsilon$, provided that $A$ and $s$ are chosen sufficiently large. This completes the inductive proof of (3.2).

Since $r=\left\lceil m^{3 / 8} / n^{1 / 8}\right\rceil$, and initially $a=m$ and $b=n$, we obtain

$$
f(m, n)=O\left(m^{3 / 4+\varepsilon^{\prime}} n^{3 / 4+\varepsilon^{\prime}}+m^{1+\varepsilon^{\prime}}+n^{1+\varepsilon^{\prime}}\right),
$$

for any $\varepsilon^{\prime}>0$. 
Step 5. For each $1 \leq i \leq k$, We compute $d\left(L_{i}, L_{i}^{\prime}\right)$ using a somewhat simpler version of the randomized algorithm described by Agarwal et al. [1]. We give a brief sketch of this variant.

5(i) Let $n_{0}$ be some sufficiently large constant. If $n_{i} \leq n_{0}$, we compute $d\left(\ell, \ell^{\prime}\right)$ for all pairs $\ell \in L_{i}, \ell^{\prime} \in L_{i}^{\prime}$, in $O\left(m_{i}\right)$ time, and return the minimum distance.

5(ii) Assume $n_{i}>n_{0}$. Choose a random subset $A \subseteq L_{i}^{\prime}$ of size $\left\lceil n_{i} / 2\right\rceil$; each subset of size $\left\lceil n_{i} / 2\right\rceil$ is chosen with equal probability.

5 (iii) Recursively compute $\delta^{\prime}=d\left(L_{i}, A\right)$.

5(iv) Partition $L_{i}$ into $\left\lceil m_{i} / n_{i}^{1 / 3}\right\rceil$ subsets, each of size at most $n_{i}^{1 / 3}$.

5 (v) For each of these subsets $W$, compute the set

$$
B^{(W)}=\left\{\ell^{\prime} \in L_{i}^{\prime}-A \mid d\left(W,\left\{\ell^{\prime}\right\}\right)<\delta^{\prime}\right\}
$$

5(vi) Compute $d\left(\ell, \ell^{\prime}\right)$ for all pairs $\ell \in W, \ell^{\prime} \in B^{(W)}$, let $\delta^{(W)}$ denote the minimum distance (put $\delta^{(W)}=+\infty$ if $B^{(W)}=\emptyset$ ), repeat this for all subsets $W$, and return $\min \left\{\delta^{\prime}, \min _{W} \delta^{(W)}\right\}$.

The correctness of the algorithm is obvious (see also [1]), so we turn to analyzing its expected running time. For a line $\ell \in L_{i}$, let

$$
B^{(\ell)}=\left\{\ell^{\prime} \in L_{i}^{\prime}-A \mid d\left(\ell, \ell^{\prime}\right)<\delta^{\prime}\right\}
$$

Using a standard probabilistic argument, it can be shown that the expected size of $B^{(\ell)}$ is $O(1)$. Since $B^{(W)}=\bigcup_{\ell \in W} B^{(\ell)}$, the expected size of $B^{(W)}$ is $O\left(n_{i}^{1 / 3}\right)$, and the expected running time of Step 5 (vi) is $O\left(\left\lceil m_{i} / n_{i}^{1 / 3}\right\rceil \cdot n_{i}^{2 / 3}\right)$.

By reversing the direction of the $z$-axis, setting $\delta=\delta^{\prime}$, and using Corollary 3.2, each $W$ can be preprocessed into a data structure of size $O\left(n_{i}^{1+\varepsilon}\right)$, for any $\varepsilon>0$, so that, for each $\ell^{\prime} \in L_{i}^{\prime}-A$, we can determine in $O\left(\log n_{i}\right)$ time whether $\ell^{\prime} \in B$. The time spent in Step $5(v)$ is thus

$$
\left\lceil\frac{m_{i}}{n_{i}^{1 / 3}}\right\rceil \cdot O\left(n_{i}^{1+\varepsilon}+n_{i} \log m_{i}\right)=O\left(m_{i} n_{i}^{2 / 3+\varepsilon}+n_{i}^{1+\varepsilon}\right),
$$

which subsumes the expected cost of Step 5(vi). Let $\varphi\left(m_{i}, n_{i}\right)$ denote the maximum expected running time for computing $d\left(L_{i}, L_{i}^{\prime}\right)$ by this algorithm, where the maximum is taken over all sets $L_{i}, L_{i}^{\prime}$ of sizes $m_{i}, n_{i}$, respectively. Then we obtain the following recurrence:

$$
\varphi\left(m_{i}, n_{i}\right) \leq \varphi\left(m_{i},\left\lceil n_{i} / 2\right\rceil\right)+O\left(m_{i} n_{i}^{2 / 3+\varepsilon}+n_{i}^{1+\varepsilon}\right),
$$

and $\varphi\left(m_{i}, 1\right)=O\left(m_{i}\right)$, whose solution is easily seen to be

$$
\varphi\left(m_{i}, n_{i}\right)=O\left(m_{i} n_{i}^{2 / 3+\varepsilon}+n_{i}^{1+\varepsilon}\right) .
$$

By the choice of the parameter $r$ in algorithm CLOSEST-PAIR, the expected time spent in Step 5 is thus

$$
\sum_{i=1}^{k} \varphi\left(m_{i}, n_{i}\right)=\sum_{i=1}^{k} O\left(m_{i} n_{i}^{2 / 3+\varepsilon}+n_{i}^{1+\varepsilon}\right)
$$




$$
\begin{aligned}
& =O\left(\left(\frac{n}{r}\right)^{2 / 3+\varepsilon} \sum_{i=1}^{k} m_{i}+\left(\frac{n}{r}\right)^{1+\varepsilon} \cdot r^{3+\varepsilon^{\prime}}\right) \\
& =O\left(m^{3 / 4+\varepsilon^{\prime}} n^{3 / 4+\varepsilon^{\prime}}+m^{1+\varepsilon^{\prime}}+n^{1+\varepsilon^{\prime}}\right) .
\end{aligned}
$$

The total expected time spent by algorithm CLOSEST-PAIR, excluding the time spent in the recursive call, is thus $O\left(m^{3 / 4+\varepsilon^{\prime}} n^{3 / 4+\varepsilon^{\prime}}+m^{1+\varepsilon^{\prime}}+n^{1+\varepsilon^{\prime}}\right)$, for any $\varepsilon^{\prime}>0$.

Recall that all lines $\ell \in L_{0}$ satisfy $d\left(\{\ell\}, L^{\prime}\right)<\delta_{0}$. Recall also that $\ell_{0}$ was chosen randomly in Step 2 . If we sort the lines $\ell \in L$ in the nondecreasing order of their distances $d\left(\{\ell\}, L^{\prime}\right)$, then the probability that $\ell$ is the $i$ th element in this list is $1 / m$, and in this case we must have $\left|L_{0}\right|<i$. Let $T(m, n)$ denote the maximum expected time for algorithm CLOSEST-PAIR to compute $d\left(L, L^{\prime}\right)$, where the maximum is taken over all sets $L, L^{\prime}$ of sizes $m$ and $n$, respectively. The arguments just given imply that

$$
T(m, n) \leq \begin{cases}c_{1} m & \text { for } n \leq n_{0}, \\ c_{2} n^{1+\varepsilon} & \text { for } n>n_{0}, \quad m \leq n^{1 / 3}, \\ \frac{1}{m} \sum_{i=0}^{m-1} T(i, n)+A\left(m^{3 / 4+\varepsilon} n^{3 / 4+\varepsilon}+m^{1+\varepsilon}+n^{1+\varepsilon}\right) \\ & \text { for } n>n_{0}, \quad m>n^{1 / 3},\end{cases}
$$

for any $\varepsilon>0$. The solution of the above recurrence is easily seen to be

$$
T(m, n) \leq B\left(m^{3 / 4+\varepsilon} n^{3 / 4+\varepsilon}+m^{1+\varepsilon} \log n+n^{1+\varepsilon}\right),
$$

for any $\varepsilon>0$ and for some constant $B=B(\varepsilon)$.

To complete the analysis, we finally show:

Lemma 3.4. Algorithm CLOSEST-PAIR computes the distance $\delta^{*}=d\left(L, L^{\prime}\right)$ correctly.

Proof. We prove the lemma by double induction on $m$ and $n$. Let $L, L^{\prime}$ be appropriate sets of lines of size $m$ and $n$, respectively. If $n<n_{0}$, then the correctness is trivial (see Step 1). If $m \leq n^{1 / 3}$ (i.e., $r=1$ ), then the algorithm is also correct, by the analysis of Step 5 given above. Suppose that $m>n^{1 / 3}$ (so $r>1$ ), and that the algorithm computes $d\left(\Lambda, \Lambda^{\prime}\right)$ correctly for all sets of lines $\Lambda$ and $\Lambda^{\prime}$ such that $|\Lambda|<m$ or $|\Lambda|=m$ and $\left|\Lambda^{\prime}\right|<n$. Since the algorithm returns the distance between a line of $L$ and a line of $L^{\prime}$, it always returns a number at least as large as $\delta^{*}=d\left(L, L^{\prime}\right)$.

If $\delta^{*}=\delta_{0}$, there is nothing to prove, because Step 7 returns the minimum of $\delta_{0}, \delta_{1}$, and $\delta_{2}$. Suppose $\delta^{*}<\delta_{0}$. Let $\ell \in L, \ell^{\prime} \in L^{\prime}$ be a pair of lines with $d\left(\ell, \ell^{\prime}\right)=\delta^{*}$. If $\ell \in L_{0}$, then $d\left(L, L^{\prime}\right)=d\left(L_{0}, L^{\prime}\right)=\delta_{2}$. Since $\left|L_{0}\right|<m$, as argued above, the algorithm computes $d\left(L_{0}, L^{\prime}\right)$ correctly, by the induction hypothesis, so $d\left(L, L^{\prime}\right)$ is also correctly computed.

If $\ell \in L_{i}$ for some $1 \leq i \leq k$, then, by construction, $\ell^{\prime} \in L_{i}^{\prime}$, and therefore $d\left(L, L^{\prime}\right)=$ $d\left(L_{i}, L_{i}^{\prime}\right)=\delta_{1}$. Since $\left|L_{i}\right| \leq m$ and $\left|L_{i}^{\prime}\right| \leq n / r<n$, the claim follows again by the induction hypothesis. This completes the proof of the lemma.

Hence, we obtain the following result: 
Theorem 3.5. Given a set $L$ of $m$ red lines and a set $L^{\prime}$ of $n$ blue lines in $\mathbb{R}^{3}$, such that all red lines lie above all blue lines, $d\left(L, L^{\prime}\right)$ can be computed by a randomized algorithm in $O\left(m^{3 / 4+\varepsilon} n^{3 / 4+\varepsilon}+m^{1+\varepsilon}+n^{1+\varepsilon}\right)$ expected time, for any $\varepsilon>0$.

Combining this result with the observations in [8], which we omit here, and which show how the width itself can be computed using algorithm CLOSEST-PAIR as a subroutine, we obtain the main result of this section.

Corollary 3.6. The width of a set of $n$ points in $\mathbb{R}^{3}$ can be computed by a randomized algorithm whose expected running time is $O\left(n^{3 / 2+\varepsilon}\right)$, for any $\varepsilon>0$.

Remark 3.7. Note that algorithm ClOSEST-PAIR and its analysis do not use the fact that $L$ and $L^{\prime}$ are sets of lines in $\mathbb{R}^{3}$, and that $d(\cdot, \cdot)$ is the Euclidean distance function. In fact, Theorem 3.5 holds in a more general setting, in which $L, L^{\prime}$, and the distance function satisfy the following properties:

(i) Each object $\ell$ in $L$ is specified by four real parameters, so that $\ell$ can be represented by a point $\varphi(\ell)$ in $\mathbb{R}^{4}$.

(ii) For any parameter $\delta>0$ and any object $\ell^{\prime} \in L^{\prime}$, a trivariate algebraic function $f_{\ell^{\prime}}\left(x_{1}, x_{2}, x_{3}\right)$ of constant degree can be defined such that, for any $\ell \in L$ with $\varphi(\ell)=\left(a_{1}, a_{2}, a_{3}, a_{4}\right), a_{4} \leq f_{\ell^{\prime}}\left(a_{1}, a_{2}, a_{3}\right)$ if and only if $d\left(\ell, \ell^{\prime}\right) \leq \delta$.

(iii) For any subset $R \subseteq L^{\prime}$ of size $r$ and $\delta>0$, the vertical decomposition of the minimization diagram of the set $\left\{f_{\ell^{\prime}} \mid \ell^{\prime} \in R\right\}$ has $O\left(r^{3+\varepsilon}\right)$ cells, for any $\varepsilon>0$.

\section{Minimum-Width Annulus}

Given a set $S=\left\{p_{1}, \ldots, p_{n}\right\}$ of $n$ points in the plane, we want to compute an annulus of the smallest width that contains $S$. That is, we want to compute two concentric circles, centered at a point $\xi$, of radii $r_{1}, r_{2}$, respectively, such that $r_{2}-r_{1}$ is minimized, subject to the constraints $r_{1} \leq d\left(\xi, p_{i}\right) \leq r_{2}$, for each $1 \leq i \leq n$ (Fig. 1).

For a given point $\mathbf{x}$, let $\omega(\mathbf{x})$ denote the smallest width of an annulus containing $S$ and centered at $\mathbf{x}$. Let $p_{\mathrm{c}}, p_{\mathrm{f}}$ be the nearest and the farthest neighbors of $\mathbf{x}$ in $S$, respectively. Then

$$
\omega(\mathbf{x})=d\left(\mathbf{x}, p_{\mathrm{f}}\right)-d\left(\mathbf{x}, p_{\mathrm{c}}\right)
$$

Let $V{ } r_{\mathrm{c}}(S)$ (resp. $V \mathrm{~V}_{\mathrm{f}}(S)$ ) denote the closest-neighbor (resp. farthest-neighbor) Voronoi diagram of $S$. Ebara et al. [15] observed that the center of a minimum-width annulus containing $S$ is a vertex of $\operatorname{Vor}_{\mathrm{c}}(S)$, a vertex of $\operatorname{Vor}_{\mathrm{f}}(S)$, or an intersection point of an edge of $\operatorname{Vor}_{\mathrm{c}}(S)$ and an edge of $\operatorname{Vor}_{\mathrm{f}}(S)$ (see also [31]). Based on this observation, they gave a rather simple $O\left(n^{2}\right)$-time algorithm for computing a minimum-width annulus, which was improved by Agarwal et al. [3] to $O\left(n^{8 / 5+\varepsilon}\right)$, and subsequently by Agarwal et al. [1] to $O\left(n^{17 / 11+\varepsilon}\right)$. Here we obtain a further improved algorithm that runs in $O\left(n^{3 / 2+\varepsilon}\right)$ expected time, for any $\varepsilon>0$, using the machinery developed in the previous section. If one is interested in minimizing the area of the annulus, instead of 


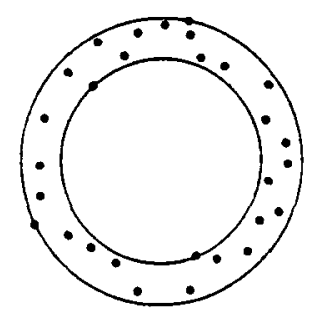

Fig. 1. A minimum-width annulus.

the width, the problem can be formulated as an instance of linear programming in $\mathbb{R}^{4}$, and therefore can be solved in $O(n)$ time, using Megiddo's algorithm [27]. See also [22] and [32] for other related results.

We first make the following observation, which was missed in the earlier treatments of the problem cited above, and which transforms the problem into a width-like problem in $\mathbb{R}^{3}$. We lift the points of $S$ to the paraboloid $z=x^{2}+y^{2}$ by the standard lifting map $(x, y) \mapsto\left(x, y, x^{2}+y^{2}\right)$. Then a circle $C$ of radius $r$ with center $(a, b)$ is mapped to the plane $C^{*}: z=2 a x+2 b y+\left(r^{2}-a^{2}-b^{2}\right)$. A point $p \in \mathbb{R}^{2}$ lies inside (resp. on, outside) $C$ if and only if its lifted image $p^{*}$ lies below (resp. on, above) the plane $C^{*}$. Thus an annulus with center $(a, b)$ and radii $r_{1}<r_{2}$ and containing $S$ is mapped to a pair of parallel planes

$$
z=2 a x+2 b y+\left(r_{1}^{2}-a^{2}-b^{2}\right)
$$

and

$$
z=2 a x+2 b y+\left(r_{2}^{2}-a^{2}-b^{2}\right),
$$

such that the image $S^{*}$ of $S$ is fully contained in the slab bounded between these two planes. In other words, the minimum-width annulus problem reduces to the problem of finding a pair of parallel planes

$$
\pi_{1}: \quad z=\kappa_{1} x+\kappa_{2} y+\kappa_{3}^{\prime} \quad \text { and } \quad \pi_{2}: \quad z=\kappa_{1} x+\kappa_{2} y+\kappa_{3}
$$

that support $S^{*}$, such that

$$
g\left(\pi_{1}, \pi_{2}\right)=\sqrt{\kappa_{3}^{\prime}+\frac{\kappa_{1}^{2}+\kappa_{2}^{2}}{4}}-\sqrt{\kappa_{3}+\frac{\kappa_{1}^{2}+\kappa_{2}^{2}}{4}}
$$

is minimized.

For a pair of (skew) lines $\ell_{1}, \ell_{2}$ in $\mathbb{R}^{3}$, we define the distance between $\ell_{1}$ and $\ell_{2}$ to be $d\left(\ell_{1}, \ell_{2}\right)=g\left(\pi_{1}, \pi_{2}\right)$, where $\pi_{1}, \pi_{2}$ are parallel planes containing $\ell_{1}, \ell_{2}$, respectively. Using the observation in [8], this width-like problem can also be formulated as the bichromatic closest line-pair problem defined in the beginning of Section 3 (i.e., given a set $L$ of $m$ lines in $\mathbb{R}^{3}$ and another set $L^{\prime}$ of $n$ lines in $\mathbb{R}^{3}$ such that all lines in $L$ lie above all lines in $L^{\prime}$, compute a closest pair between $L$ and $L^{\prime}$ ), except that we use the distance function just described. An explicit expression for $d\left(\ell, \ell^{\prime}\right)$ can be obtained as 
follows. Let the equations defining $\ell, \ell^{\prime}$ be, as in Section 3 ,

$$
\begin{aligned}
& \ell: \quad y=a_{1} x+a_{3}, \quad z=a_{2} x+a_{4} \text {, } \\
& \ell^{\prime}: \quad y=b_{1} x+b_{3}, \quad z=b_{2} x+b_{4} \text {. }
\end{aligned}
$$

The direction of the common normal to both lines is

$$
\mathbf{n}=\left(a_{1} b_{2}-a_{2} b_{1}, a_{2}-b_{2},-\left(a_{1}-b_{1}\right)\right),
$$

and the planes orthogonal to $\mathbf{n}$ and containing $\ell, \ell^{\prime}$, respectively, are

$$
\left(\mathbf{x}-\left(0, a_{3}, a_{4}\right)\right) \cdot \mathbf{n}=0 \quad \text { and } \quad\left(\mathbf{x}-\left(0, b_{3}, b_{4}\right)\right) \cdot \mathbf{n}=0 \text {, }
$$

or

$$
z=\frac{a_{1} b_{2}-a_{2} b_{1}}{a_{1}-b_{1}} x+\frac{a_{2}-b_{2}}{a_{1}-b_{1}} y-\frac{a_{3}\left(a_{2}-b_{2}\right)-a_{4}\left(a_{1}-b_{1}\right)}{a_{1}-b_{1}}
$$

and

$$
z=\frac{a_{1} b_{2}-a_{2} b_{1}}{a_{1}-b_{1}} x+\frac{a_{2}-b_{2}}{a_{1}-b_{1}} y-\frac{b_{3}\left(a_{2}-b_{2}\right)-b_{4}\left(a_{1}-b_{1}\right)}{a_{1}-b_{1}} .
$$

Plugging this into (4.1), we thus can write

$$
d\left(\ell, \ell^{\prime}\right)=\sqrt{\mu a_{3}+a_{4}+v}-\sqrt{\mu b_{3}+b_{4}+v},
$$

where $\mu$ and $v$ depend only on $a_{1}, a_{2}, b_{1}, b_{2}$.

In order to apply Algorithm CLOSEST-PAIR, we need to prove an appropriate variant of Lemma 3.1. First, we note that if $\ell^{\prime}$ is fixed, then $d\left(\ell, \ell^{\prime}\right)$ is defined whenever the $\xi_{1}$-coordinate of $\ell$ is different than that of $\ell^{\prime}$. (It is easily verified that, for the lines $\ell, \ell^{\prime}$ arising in our analysis, the arguments of the square roots in (4.1) are never negative.) In this case $d\left(\ell, \ell^{\prime}\right)$ monotonically increases with $a_{4}$, which implies that the appropriate variant of the functions $f_{\ell^{\prime}}$ is well defined (whenever the $\xi_{1}$-coordinates of $\ell$ and of $\ell^{\prime}$ are different). To establish the second part of the lemma, for a fixed parameter $\delta$, let $\ell_{1}^{\prime}$, $\ell_{2}^{\prime}$ be two given lines, let $\xi_{1}$ and $\xi_{2}$ be fixed (with $\xi_{1}$ different from the $\xi_{1}$-coordinates of $\ell_{1}^{\prime}$ and of $\ell_{2}^{\prime}$ ), and consider the equation

$$
f_{\ell_{1}^{\prime}}\left(\xi_{1}, \xi_{2}, \xi_{3}\right)=f_{\ell_{2}^{\prime}}\left(\xi_{1}, \xi_{2}, \xi_{3}\right)
$$

We seek a line $\ell$ that lies above $\ell_{1}^{\prime}$ and $\ell_{2}^{\prime}$ and satisfies $d\left(\ell, \ell_{1}^{\prime}\right)=d\left(\ell, \ell_{2}^{\prime}\right)=\delta$. This can be written as

$$
\begin{aligned}
& \sqrt{\mu_{1} \xi_{3}+\xi_{4}+v_{1}}=\sqrt{\mu_{1} b_{3}^{(1)}+b_{4}^{(1)}+v_{1}}+\delta \\
& \sqrt{\mu_{2} \xi_{3}+\xi_{4}+v_{2}}=\sqrt{\mu_{2} b_{3}^{(2)}+b_{4}^{(2)}+v_{2}}+\delta
\end{aligned}
$$

where $b^{(1)}, b^{(2)}$ are the 4-tuples defining $\ell_{1}^{\prime}, \ell_{2}^{\prime}$, respectively, and $\mu_{1}, v_{1}, \mu_{2}, v_{2}$ are independent of $\xi_{3}, \xi_{4}$. We thus get a linear system of equations in $\xi_{3}$, $\xi_{4}$, which can be 
easily reduced to a linear equation in $\xi_{3}$ of the form $\left(\mu_{1}-\mu_{2}\right) \xi_{3}=\alpha$. This shows that (4.2) has a unique solution, unless $\mu_{1}=\mu_{2}$, or

$$
\frac{\xi_{2}-b_{2}^{(1)}}{\xi_{1}-b_{1}^{(1)}}=\frac{\xi_{2}-b_{2}^{(2)}}{\xi_{1}-b_{1}^{(2)}},
$$

which is the equation of a line in the $\xi_{1} \xi_{2}$-plane.

We have thus shown that conditions (F1) and (F2) of Section 2, and therefore the conditions of Remark 3.6, are satisfied in this case too, so the algorithms and the analysis of the preceding section apply here as well. We thus obtain the following result.

Theorem 4.1. An annulus of smallest width that contains a given set $S$ of $n$ points in the plane can be computed by a randomized algorithm in $O\left(n^{3 / 2+\varepsilon}\right)$ expected time, for any $\varepsilon>0$.

\section{Biggest Stick in a Polygon}

The biggest-stick problem is to find the longest segment that can be placed inside a simple $n$-gon $P$ in the plane. The problem was posed by McKenna [25], who gave an $O\left(n^{2}\right)$-time solution. Chazelle and Sharir gave an $O\left(n^{1.9999}\right)$-time algorithm [11], which was improved by Agarwal et al. [1] to $O\left(n^{17 / 11+\varepsilon}\right)$, for any $\varepsilon>0$; see also [3] for an intermediate bound. If the endpoints of the segment are restricted to be at vertices of $P$, the problem becomes considerably easier, and can be solved in $O\left(n \log ^{3} n\right)$ time [4].

Following the same idea as in [3], we use a divide-and-conquer approach. Partition $P$ into two simple polygons $P_{1}, P_{2}$ by a chord $c$, so that each of $P_{1}, P_{2}$ has at most $2 n / 3$ vertices (Fig. 2). Let $l_{c}$ be the line passing through $c$. We recursively compute the longest segment that can be placed within $P_{1}$ or within $P_{2}$. The merge step requires computing the longest segment having one endpoint in $P_{1}$ and the other in $P_{2}$. An easy perturbation argument shows that such a longest segment has to touch two vertices of $P$, say, $v_{1}, v_{2}$. The difficult case is when $v_{1} \in P_{1}$ and $v_{2} \in P_{2}$ (the case in which both $v_{1}$ and $v_{2}$ belong to the same subpolygon is easy, because there can be only $O(n)$ such

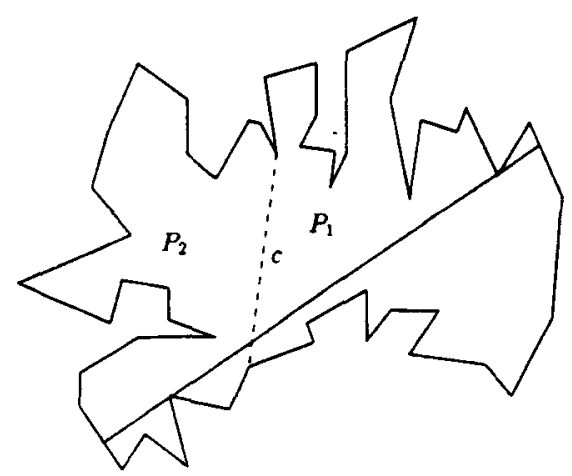

Fig. 2. Biggest stick in a polygon. 


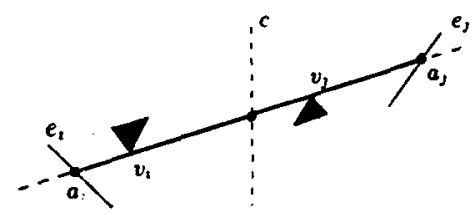

Fig. 3. Illustration of the distance function $d\left(\ell_{i}, \ell_{j}\right)$.

pairs of vertices-see [3]). Agarwal et al. [3] showed that finding such a segment can be reduced to the following problem: We have a set $L_{1}$ of $a$ lines in the plane, where each line $\ell_{i} \in L_{1}$ is dual to some vertex $v_{i}$ of $P_{1}$ and has an edge $e_{i} \in P_{1}$ associated with it. Similarly, we have another set $L_{2}$ of $b$ lines in the plane, where each line $\ell_{j} \in L_{2}$ is dual to some vertex $v_{j}$ of $P_{2}$ and has an edge $e_{j}$ of $P_{2}$ associated with it. $L_{1}$ and $L_{2}$ satisfy the following property: For any pair $\ell_{i} \in L_{1}, \ell_{j} \in L_{2}$, the segment $g_{i j}=a_{i} a_{j}$ lies inside $P$, where $a_{i}$ (resp. $a_{j}$ ) is the intersection point of $e_{i}$ (resp. $e_{j}$ ) with the line passing through $v_{i}$ and $v_{j}$; see Fig. 3. The goal is to compute the longest segment $g_{i j}$, over all pairs $\ell_{i} \in L_{1}, \ell_{j} \in L_{2}$. If we define the distance function $d\left(\ell_{i}, \ell_{j}\right)$ as the length of the segment $g_{i j}$, then the goal is to compute a farthest pair in $L_{1} \times L_{2}$. The reduction of the original subproblem yields many instances of this problem, and the biggest stick that crosses the chord $c$ is the largest of all longest segments $g_{i j}$, over all instances.

We next show that $L_{1}, L_{2}$ can be parametrized by four real parameters (two for the coordinates of the associated vertex of $P$ and two for the coefficients of the line containing the associated edge), so that the setup of Remark 3.6 arises here as well. In more detail, we first assume, without loss of generality, that $c$ lies on the $y$-axis, and that, locally near $c, P_{1}$ lies to the left of $c$ and $P_{2}$ lies to the right. Each line $\ell_{i} \in L_{1}$, dual to a vertex $v_{i}$ (where, as follows from the analysis of [3], $v_{i}$ lies in the half-plane $x<0)$, and associated with an edge $e_{i}$, is mapped to a point $\left(a_{1}, a_{2}, a_{3}, a_{4}\right) \in \mathbb{R}^{4}$, where $a_{1}, a_{2}$ are the coordinates of the vertex $v_{i}$ and where the equation of the line containing $e_{i}$ is $y=a_{3} x+a_{4}$ (we assume, without loss of generality, that no such line is vertical). Let $w$ be a real parameter. For each line $\ell_{j} \in L_{2}$, dual to a vertex $v_{j}$ (lying in the halfplane $x>0$; see [3]), and associated with an edge $e_{j}$, we define a trivariate function $x_{4}=f_{j}\left(x_{1}, x_{2}, x_{3}\right)$, as follows. Given $\left(x_{1}, x_{2}, x_{3}, x_{4}\right)$, with $x_{1}<0$, let $v$ be the point $\left(x_{1}, x_{2}\right)$, let $\ell$ be the line $y=x_{3} x+x_{4}$, and let $a$ (resp. $b$ ) be the intersection point of the line containing $e_{j}$ (resp. $\ell$ ) with the line $\lambda$ passing through the points $v_{j}$ and $v$. If both $a$ and $b$ uniquely exist, then we put $x_{4}=f_{j}\left(x_{1}, x_{2}, x_{3}\right)$ if $d(a, b)=w$. If $a$ does not exist uniquely, then $f_{j}$ is undefined. If $a$ uniquely exists and $x_{1}, x_{2}, x_{3}$ are such that $\ell$ and $\lambda$ are parallel, then $f_{j}\left(x_{1}, x_{2}, x_{3}\right)$ is the unique $x_{4}$ for which $\ell$ and $\lambda$ coincide. The functions $f_{j}$ are easily seen to be algebraic, of constant maximum degree. They are all undefined for $x_{1} \geq 0$, so the functions of $\mathcal{F}$ are defined only on a corresponding half-space of $\mathbb{R}^{4}$. We leave it to the reader to verify that (a straightforward variant of ) the machinery developed in earlier sections is applicable in this case too.

Lemma 5.1. The set $\mathcal{F}=\left\{f_{j} \mid \ell_{j} \in L_{2}\right\}$ satisfies (an appropriately modified variant of) the assumptions (F1) and (F2). 


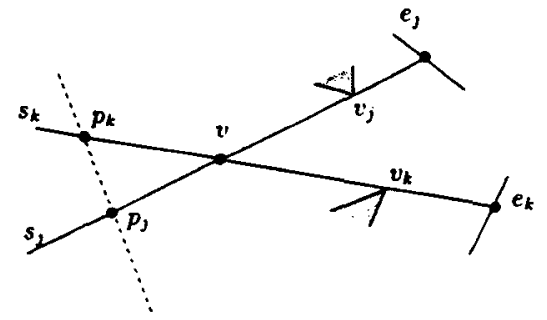

Fig. 4. Illustration of the case $f_{j}\left(x_{1}, x_{2}, x_{3}\right)=f_{k}\left(x_{1}, x_{2}, x_{3}\right)$.

Proof. To establish (F1), we need to show that, for a given line $\ell_{j} \in L_{2}$ and for fixed $x_{1}, x_{2}$, with $x_{1}<0$, the value $f_{j}\left(x_{1}, x_{2}, x_{3}\right)$ is defined for all $x_{3}$, except when $\left(x_{1}, x_{2}\right)$ lies on any of a constant number of algebraic arcs of constant maximum degree. Since the point $\left(x_{1}, x_{2}\right)$ is fixed, the line $\lambda$ passing through $v_{j}$ and $\left(x_{1}, x_{2}\right)$ is also fixed. If $\lambda$ is parallel to $e_{j}$, then $f_{j}\left(x_{1}, x_{2}, x_{3}\right)$ is undefined for all $x_{3}$. The locus of points $\left(x_{1}, x_{2}\right)$ where this condition holds is easily seen to be a line in the $x_{1} x_{2}$-plane. Otherwise, there exists a unique point $p$ on $\lambda$ at distance $w$ from the intersection of $\lambda$ with the line containing $e_{j}$ and lying to the left of this intersection, and for any $x_{3}$, there is a unique line $y=x_{3} x+x_{4}$ passing through $p$, so $f_{j}\left(x_{1}, x_{2}, x_{3}\right)$ is defined for all $x_{3}$.

Next, to establish (F2), we need to show that, for two given lines $\ell_{j}, \ell_{k} \in L_{2}$, and for any fixed $\left(x_{1}, x_{2}\right)$ not lying on any of the arcs where $f_{j}$ or $f_{k}$ is undefined or on a few additional arcs, detailed below, the equation

$$
f_{j}\left(x_{1}, x_{2}, x_{3}\right)=f_{k}\left(x_{1}, x_{2}, x_{3}\right)
$$

has at most one solution $x_{3}$ (Fig. 4). Assume that both functions are defined over $\left(x_{1}, x_{2}\right) \times$ $\mathbb{R}$. Suppose that $x_{3}$ is a solution of this equation, and that $x_{4}=f_{j}\left(x_{1}, x_{2}, x_{3}\right)$. Let $v$ be the fixed point $\left(x_{1}, x_{2}\right)$. Let $s_{j}$ (resp. $s_{k}$ ) be the unique segment of length $w$ emanating from a point on $e_{j}$ (resp. on $e_{k}$ ) to the left, and contained in the line passing through $v_{j}$ and $v$ (resp. through $v_{k}$ and $v$ ). Let $p_{j}$ (resp. $p_{k}$ ) be the left endpoint of $s_{j}$ (resp. $s_{k}$ ). Then the line $y=x_{3} x+x_{4}$ is the unique line connecting $p_{j}$ and $p_{k}$. This is true as long as either $p_{j}$ or $p_{k}$ is different from $v$. (If $p_{j}=p_{k}=v$, then $y=x_{3} x+x_{4}$ is undefined.) In other words, to be on the safe side, we do not want $v$ to have the property that the length of one of the segments connecting $v$ to $e_{j}$ through $v_{j}$ or to $e_{k}$ through $v_{k}$ is equal to $w$. This implies that, as long as $v$ does not lie on one of two corresponding algebraic curves (these curves are of degree 4, and are known as "conchoids of Nicomedes" [23]), the above equation has a unique solution.

This completes the proof of the lemma.

In view of the above lemma, the conditions of Remark 3.7 are satisfied here, so we can apply a variant of algorithm CLOSEST-PAIR to find a farthest pair in $L_{1}$ and $L_{2}$. Omitting the further, easy and technical details on how the full problem is solved using algorithm CLOSEST-PAIR (these details can be found in [1] and [3]), we obtain the following result:

Theorem 5.2. The longest segment that can be placed inside a given simple $n$-gon $P$ can be computed by a randomized algorithm in $O\left(n^{3 / 2+\varepsilon}\right)$ expected time, for any $\varepsilon>0$. 


\section{Conclusion}

In this paper we have presented efficient randomized algorithms for three geometric optimization problems. These algorithms are not only asymptotically faster than the previously best known algorithms, but they are simpler as well. Our technique also yields simpler algorithms for other geometric optimization problems, such as computing a closest line pair in 3-space [8], [28].

We conclude this paper by mentioning some open problems:

(i) Can our algorithms be derandomized without affecting their running time significantly?

(ii) Computing a minimum-width annulus containing a set $S$ of points in the plane is equivalent to fitting a circle $C$ through $S$, such that the maximum distance between the points of $S$ and $C$ is minimized. However, in some applications, such as computational metrology [16], [34], the interest is in minimizing the sum of squares of distances between $C$ and the points of $S$. We are not aware of any efficient algorithm for this problem.

(iii) There are various interesting extensions of the biggest-stick problem, which are worth pursuing. For example, given a simple polygon $P$ and a triangle $T$, is there a subquadratic algorithm that determines whether $T$ can be placed inside $P$, allowing both translations and rotations? A quadratic algorithm for a special case of this problem when $P$ is a convex polygon was given by Chazelle [6]. Another related problem is: Given a segment $e$ and a polygon $P$ with holes, determine, in subquadratic time, whether $e$ can be placed inside $P$, using translations and rotations. A quadratic solution can be obtained by modifying Welzl's algorithm [33] for computing the visibility graph of $P$.

\section{References}

1. P. Agarwal, B. Aronov, and M. Sharir, Computing lower envelopes in four dimensions with applications, Proc. 10th Annual Symp. on Computational Geometry, 1994, pp. 348-358.

2. P. Agarwal, O. Schwarzkopf, and M. Sharir, The overlay of lower envelopes in three dimensions and its applications, Proc. 11th Annual Symp. on Computational Geometry, 1995, pp. 182-189.

3. P. Agarwal, M. Sharir, and S. Toledo, New applications of parametric searching in computational geometry, J. Algorithms 17 (1994), 292-318.

4. A. Aggarwal and S. Suri, The biggest diagonal in a simple polygon, Inform. Process. Lett. 35 (1990), 13-18.

5. H. Brönnimann and B. Chazelle, Optimal slope selection via cuttings, Proc. 6th Canadian Conf. on Computational Geometry, 1994, pp. 99-103.

6. B. Chazelle, The polygon containment problem, in: Advances in Computing Research, Vol. I: Computational Geometry (F. Preparata, ed.), JAI Press, Greenwich, CT, 1993, pp. 1-33.

7. B. Chazelle, H. Edelsbrunner, L. Guibas, and M. Sharir, A singly exponential stratification scheme for real semi-algebraic varieties and its applications, Proc. 16th Internat. Colloq. on Automata, Languages and Programming, 1989, pp. 179-192. Lecture Notes in Computer Science, vol. 371, Springer-Verlag, Berlin. (Also in Theoret. Comput. Sci. 84 (1991), 77-105.)

8. B. Chazelle, H. Edelsbrunner, L. Guibas, and M. Sharir, Diameter, width, closest line pair, and parametric searching, Discrete Comput. Geom. 10 (1993), 183-196.

9. B. Chazelle and M. Sharir, An algorithm for generalized point location and its applications, J. Symbolic Comput. 10 (1990), 281-309. 
10. K. Clarkson, H. Edelsbrunner, L. Guibas, M. Sharir, and E. Welzl, Combinatorial complexity bounds for arrangements of curves and spheres, Discrete Comput. Geom. 5 (1990), 99-160.

11. K. Clarkson and P. Shor, Applications of random sampling in computational geometry, Il, Discrete Comput. Geom. 4 (1989), 387-421.

12. M. de Berg, K. Dobrindt, and O. Schwarzkopf, On lazy randomized incremental construction, Discrete Comput. Geom. 14 (1995), 261-286.

13. M. de Berg, D. Halperin, and L. Guibas, Vertical decomposition for triangles in 3-space, Proc. 10th Annual Symp. on Computational Geometry, 1994, pp. 1-10.

14. M. Dillencourt, D. Mount, and N. Netanyahu, A randomized algorithm for slope selection, Internat. J. Comput. Geom. Appl. 2 (1992), 1-27.

15. H. Ebara, N. Fukuyama, H. Nakano, and Y. Nakanishi, Roundness algorithms using the Voronoi diagrams, First Canadian Conf. on Computational Geometry, 1989.

16. A. Forbes, Generalized regression problems in metrology, Numer. Algorithms 5 (1993), 523-533.

17. D. Halperin and M. Sharir, New bounds for lower envelopes in three dimensions, with applications to visibility in terrains, Discrete Comput. Geom. 12 (1994), 313-326.

18. D. Haussler and E. Welzl, $\varepsilon$-nets and simplex range queries, Discrete Comput. Geom. 2 (1987), 127-151.

19. M. Houle and G. Toussaint, Computing the width of a set, IEEE Trans. Pattern Anal. Mach. Intell. 5 (1988), 761-765.

20. M. Katz and M. Sharir, Optimal slope selection via expanders, Inform. Process. Lett. 47 (1993), 115-122.

21. M. Katz and M. Sharir, An expander-based approach to geometric optimization, Proc. 9th Annual Symp. on Computational Geometry, 1993, pp. 198-207. (Also to appear in SIAM J. Comput.)

22. V. B. Le and D. T. Lee, Out-of-roundness problem revisited, IEEE Trans. Pattern Anal. Mach. Intell. 13 (1991), 217-223

23. E. H. Lockwood, A Book of Curves, Cambridge Unjversity Press, Cambridge, 1967.

24. J. Matoušek, Randomized optimal algorithm for slope selection, Inform. Process. Lett. 39 (1991), 183-187.

25. M. McKenna, The biggest stick problem, First Computational Geometry Day, New York University, New York, 1986.

26. N. Megiddo, Applying parallel computation algorithms in the design of serial algorithms, J. Assoc. Comput. Mach. 30 (1983), 852-865.

27. N. Megiddo, Linear programming in linear time when the dimension is fixed, J. Assoc. Comput. Mach. 31 (1984), 114-127.

28. M. Pellegrini, On collision-free placement of simplices and the closest pair of lines in 3-space, SIAM J. Comput. 23 (1994), 133-153.

29. M. Sharir, Almost tight upper bounds for lower envelopes in higher dimensions, Discrete Comput. Geom. 12 (1994), 327-345.

30. M. Sharir and P. Agarwal, Davenport-Schinzel Sequences and Their Geometric Applications, Cambridge University Press, Cambridge, 1995.

31. M. Smid and R. Janardan, On the Width and Roundness of a Set of Points in the Plane, Tech. Rept. MPI-I-94-111, Max-Planck-Institut für Informatik, Saarbrücken, 1994.

32. K. Swanson, An optimal algorithm for roundness determination on convex polygons, Proc. 3rd Workshop on Algorithms Data Structures, 1993, pp. 601-609.

33. E. Welzl, Constructing the visibility graph for $n$ line segments in $O\left(n^{2}\right)$ time, Inform. Process. Letters 20 , 167-171.

34. G. Wilfong, private communication.

Received June 15, 1995, and in revised form November 20, 1995. 